\title{
Aplicação de Análise de Valor em Simulação de Alternativas de Projetos Industriais
}

\author{
FERNANDO MARTINELI LOUREIRO \\ PAULO JOSÉ DE FREITAS FILHO
}

Universidade Federal de Santa Catarina

Programa de Pós-Graduação em Eng. de Produção

Caixa Postal 476 - Florianópolis, SC - CEP 88040-900

Palavras chaves: análise de valor, projeto de experimento, simulação.

\section{RESUMO}

O presente trabalho vis a apresentar o uso da Análise de Valor em simulação de processos industriais. objetivando uma resposta mais rápida e com maior segurança, na fase de tomada de decisão. 


\section{Introdução}

Face ao aumento da competitividade do mercado, as empresas são forçadas, cada vez mais, a fazer investimentos em suas plantas industriais, visando o aumento de produtividade. A utilização de ferramentas específicas à análise de tomada de decisão proporcionará uma base mais segura para a consecução dos objetivos. Aqui procurar-se-á demonstrar que é possivel a integração de algumas ferramentas de auxílio à tomada de decisão, tais como simulação, análise de valor e planejamento de experimentos, em ações de melhoria ou de reprojeto de processos.

\section{Os Riscos da}

\section{Mudança}

Nos processos de aperfeiçoamento, seja através de melhoria ou de reengenharia, as ações são conduzidas através de análise de alternativas. Contudo, incertezas se relacionam a cada uma destas, gerando de forma associada, riscos ao processo de decisório. Portanto, existem duas maneiras de se lidar com estes riscos: evitando o risco, através da manutenção do status-quo; ou tomando um risco calculado, feito através da avaliação das alternativas disponíveis. Obviamente, quanto mais profunda for a mudança, maior será o risco e a necessidade de utilização de ferramentas auxiliares ao processo de tomada de decisão.

\section{A Simulação}

A simulação é uma ferramenta largamente utilizada há vários anos, capaz de auxiliar o processo de tomada de decisão. É empregada para predizer o comportamento de sistemas complexos, através do cálculo das interações entre subsistemas. Sua principal vantagem é não interferir diretamente no chão de fábrica, evitando assim problemas relacionados a implantação de propostas equivocadas.

\section{Análise de Valor}

A análise de valor aqui usada em processos, pode ser concebida como a aplicação sistemática de técnicas reconhecidas que: a) identificam a função de processo; b) estabelecem um valor para aquela função; c) objetivam prover tal função ao menor custo total sem degradação do mesmo.

\section{Sinergia Gerada pelo Uso Concomitante de Análise de Valor e Simulação}

O uso combinado de análise de valor e de simulação, não somente é possível, como também possui força sinérgica, pois uma ferramenta se beneficia da utilização da outra; i.e., a utilização da simulação é auxiliada pela análise de valor durante a geração de alternativas, possibilitando um refinamento das alternativas colocadas, ao mesmo tempo que permite incorporar parâmetros necessários à análise; e mutatis mutandis, o uso de análise de valor se aproveita da simulação durante a análise das alternativas, diminuindo a chance de eliminação de propostas viáveis (erro ), ao mesmo tempo que permite agregar mais elementos à análise de alternativas, sem incorrer no risco gerado pelo excesso de simplificação. 


\section{Metodologia Proposta}

A metodologia, ora proposta, procura criar uma estrutura de utilização das ferramentas de suporte à tomada de decisão, visando a integração das mesmas, através de um fluxo de procedimentos. A metodologia proposta pode ser visualizada na figura 1 . do uso de fatoriais fracionários, procura reduzir o número de experimentos necessários à validação estatística do modelo. Os conceitos oriundos da metodologia Taguchi são considerados nas fases de projeto de experimentos, através do uso de arranjos ortogonais, e de análise de resultados, pelo uso da análise de variância (ANOVA).

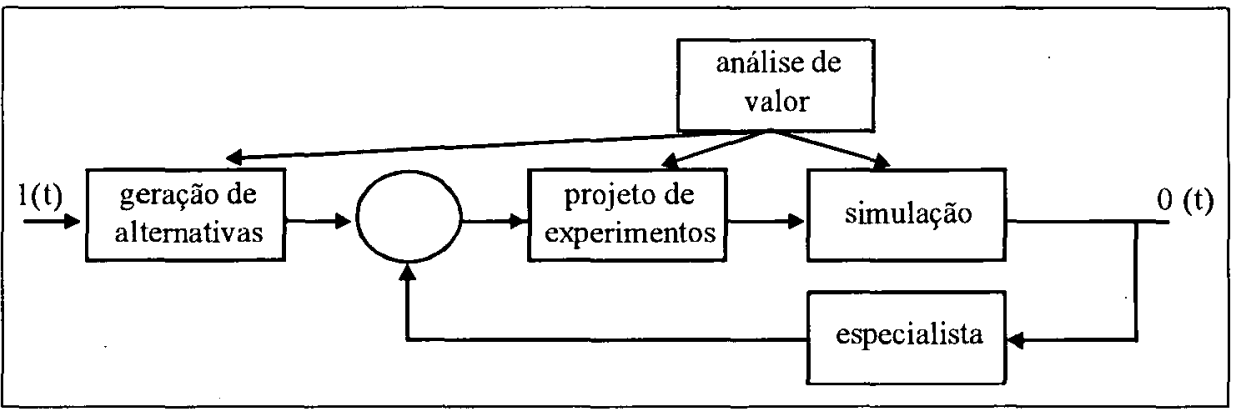

Figura 1. Metodologia proposta

O estudo se baseia na análise das alternativas propostas para a melhoria do processo. Parte-se de uma filtragem inicial das propostas colocadas, passando então para o projeto do experimento, que é feito com o objetivo de preparar os dados para a simulação. Terminada a fase de simulação, o especialista, ou o sistema especialista, verifica, com base nas respostas produzidas, se há necessidade de continuar o processo (feedback), ou se ainda é possivel fazer novas alterações.

A seguir são descritos, de forma mais detalhada, os blocos da figura 1 .

a) Geração de alternativas - feita com base na experiência do grupo envolvido com o processo de mudança. As alternativas geradas, feitas geralmente em sessões de brainstorming, são analisadas, selecionando aquelas que o grupo considere como viáveis.

b) Projeto de experimentos - feito através c) Simulação - visa analisar o reflexo da implantação de propostas alternativas no sistema produtivo.

d) Especialista - şeu uso é necessário, uma vez que a simulação não é ferramenta de otimização. Visa fornecer ao processo de tomada de decisão um banco de conhecimento que irá guiar a análise. $O$ especialista tem, portanto, o dever de: i) verificar se o modelo se adequa a realidade; ii) propor e verificar altemativas; iii) analisar as respostas, realimentando o processo. Cabe ainda ao especialista interromper o processo, escolhendo a altemativa a ser implantada, ou decidindo pela não existência de qualquer alternativa possivel ao sistema;

e) Análise de valor - visa fornecer subsidios à análise e tomada de decisão, como, por exemplo, na verificação da viabilidade de alternativas. O seu uso também se fará necessário durante a fase de elaboração do modelo 
de simulação, através do fornecimento de parâmetros necessários à análise. Procurarse-á trabalhar aqui com parâmetros relacionados a custeio por atividade, bem como parâmetros relacionados a análise de fluxo, como porexemplo MCE (Manufacturing Cycle Efficiecy). Como metodologia de análise de valor, sugere-se aquela formulada pelo Departamento de Defesa dos Estados Unidos (DOD Handbook 5010 8-4, 1968) (figura 2).

\section{Exemplo}

Uma indústria fabricante de dois produtos, acredita que reduzindo o preço de seus produtos conseguirá se inserir no mercado comprador perfeito, i.e. todos os produtos produzidos serão vendidos. Portanto os diretores decidiram promover alterações no processo produtivo. Para isto reuniram várias pessoas relacionadas à produção $\mathrm{e}$ à engenharia para juntos elaborarem propostas de alterações.

Seguindo a metodologia da figura 2 , ficou estabelecido que: estariam eles estudando o processo de produção dos produtos 1 e 2 , apurando como o sistema atual funciona, o quanto custa e qual seriam os custos alvo para os produtos. Na fase de especulação, várias alternativas foram sugeridas, tendo sido selecionadas as seguintes: a) variação no mix; b) compra de mais uma máquina para os subprocessos 3 e/ou 4 (vide figura 3), duplicando assim a capacidade.

O sistema de custeio foi dividido em: a) custo de fabricação e função do tempo em processo; b) material direto: CR $\$ 30,00$ para o produto 1 e CR $\$ 20,00$ par o produto 2 ; c) overhead: considerando o nível tecnológico dos produtos como iguais, o custo foi considerado igual para cada unidade.

No custo de fabricação foram considerados: a) custo de aquisição: CR \$ 4.000.000,00 para cada máquina; b) ajustamento ao custo corrente, devido às taxas inflacionárias: $6 \%$ ao ano; c) custo de financiamento: juros de $9 \%$ ao ano, medidos em relação ao valor médio da máquina.

Os custos alvo determinados foram: CR $\$ 1.800,00$ (produto A); CR $\$ 1.200,00$ (produto B).

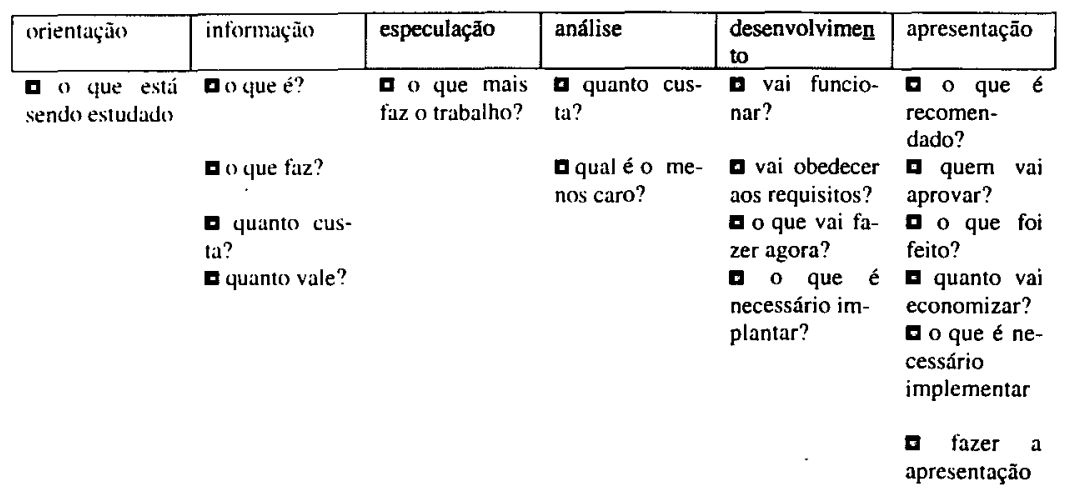

áreas de utilização da simulação

Figura 2. Plano de trabalho, conforme DOD Handbook 5010 8-4, 1968. 


\begin{tabular}{|c|c|c|c|c|c|c|c|c|c|}
\hline \multirow{3}{*}{$\exp$} & \multirow[t]{3}{*}{$\operatorname{mix}(\%)$} & \multirow[t]{3}{*}{$\begin{array}{l}\text { Fatores } \\
\text { máq.(3) }\end{array}$} & \multirow[t]{3}{*}{ máq.(4) } & \multicolumn{2}{|c|}{ Custo } & \multicolumn{2}{|c|}{ MCE } & \multicolumn{2}{|c|}{ Quantidade } \\
\hline & & & & \multicolumn{2}{|c|}{ Produto } & \multicolumn{2}{|c|}{ Produto } & \multicolumn{2}{|c|}{ Produto } \\
\hline & & & & 1 & 2 & 1 & 2 & 1 & 2 \\
\hline 1 & $50 / 50$ & 1 & 1 & 2697.60 & 1985.10 & 0.114 & 0.132 & 22 & 8 \\
\hline 2 & $50 \times 50$ & F & & ming & & & & 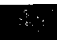 & \\
\hline 3 & $50 / 50$ & 2 & 1 & 2912.40 & 2071.50 & 0.120 & 0.146 & 21 & 16 \\
\hline 4 & $50 / 50$ & 2 & 2 & 2503.90 & 1760.40 & 0.210 & 0.200 & 30 & 19 \\
\hline 5 & $70 / 30$ & 1 & 1 & 2938.20 & 2065.90 & 0.107 & 0.210 & 25 & 4 \\
\hline 6 & $70 / 30$ & l & 2 & 2534.30 & 1505.70 & 0.165 & 0.238 & 31 & 16 \\
\hline 7 & $70 / 30$ & 2 & 1 & 3327.20 & 2082.40 & 0.110 & 0.179 & 26 & 11 \\
\hline 8 & $70 / 30$ & 2 & 2 & 2864.10 & 2034.10 & 0.214 & 0.248 & 42 & 8 \\
\hline
\end{tabular}

Tabela 1. Fatores, niveis do projeto de experimentos e resultados da simulação

Observaçōes:

a) o mix se refere, respectivamente, à fabricação dos produtos $\mathrm{A}$ e $\mathrm{B}$; os fatores referem-se, respectivamente, às quantidades de máquinas instaladas nos subprocessos 3 e 4;

b) os valores de custo são dados em CR\$/ unidade, $\mathrm{e}$ as quantidades em unidade;

c) $\mathrm{O}$ experimento 1 se refere às condições iniciais e oxperimento 2 ao de melhor resultado;

d) MCE é dado pela fórmula:

$\mathrm{MCE}=$ tempo total em processo lead time.

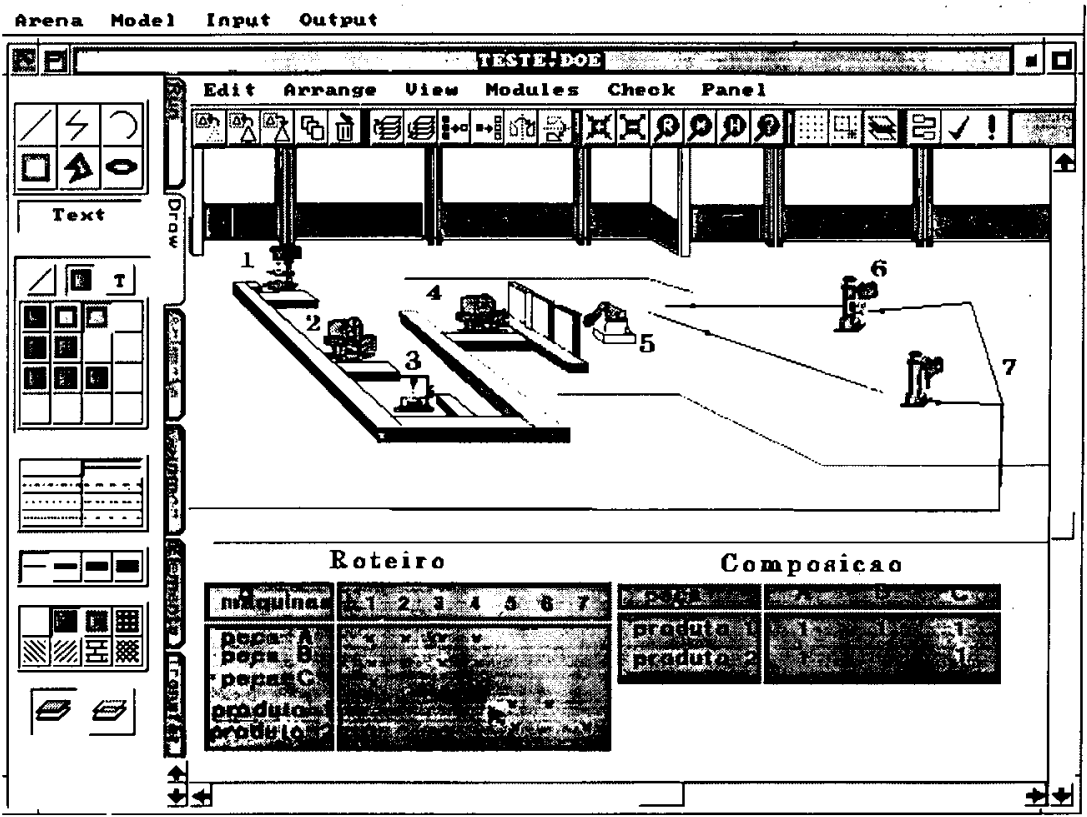

Figura 3. Planta das unidades de fabricação. Os números se relacionam aos subprocessos. Os quadros se referem ao roteiro de fabricação dos produtos e das peças, e à composição dos produtos. 


\section{Conclusão}

Os dados obtidos, conforme tabela 2 , revelaram que, revelou-se menos importante para o desempenho do processo. Contribuiu, em termos de custo, para o aumento dos mesmos. Com relação ao índice MCE, contribuiu para um aumento em ambos os produtos, o que era

\begin{tabular}{|c|c|c|c|c|c|}
\hline \multirow{2}{*}{ Fator } & \multirow[b]{2}{*}{ df: } & \multicolumn{2}{|c|}{ Cústo } & \multicolumn{2}{|c|}{ MCE: } \\
\hline & & Produto 1 & Produto 2 & Produto 1 & Produto 2 \\
\hline mix & 1 & 26,77 & $-1,31$ & 1,20 & 28,10 \\
\hline máquina 3 & 1 & 22,12 & 17,15 & 1,20 & $-1,25$ \\
\hline e & 1 & & & & \\
\hline máquina 4 & 1 & 48,19 & 50,99 & 88,52 & 57,60 \\
\hline $\mathbf{e}$ & 1 & & & & \\
\hline e & 1 & & & & \\
\hline ERRO & 4 & 2,93 & 33,17 & 9.08 & 15,55 \\
\hline
\end{tabular}

Tahcla 2. Resultado da análise de variância (em \%)

após à execução da ANOVA, o fator que mais contribuiu para a diminuição dos custos foi o fator relacionado com a máquina 4 (48,19\% para o produto 1 e $50,99 \%$ para o produto 2). Ainda, a compra de nova máquina para o subprocesso 4 se mostra o fator mais importante para o aumento de $\operatorname{MCE}(88,52 \%$ para o produto 1 e 57,60 para o produto 2). Isto indica que a capacidade do subprocesso 4 é a restrição mais importante do processo, sendo, portanto, o gargalo do sistema.

Quanto aos outros fatores, podemos identificar que:

a) a mudança do mix não afetou significantemente o processo, e ainda contribuiu para o aumentos dos custos de ambos os produtos; portanto, não se indica uma alteração do mix atual, observando apenas critérios relacionados à produção;

b) o fator relacionado com a compra de mais uma máquina para o subprocesso 3 , óbvio, uma vez que aumentou a capacidade do processo; de qualquer forma não foi um aumento significativo e, portanto, não se indica qualquer alteração para a capacidade do subprocesso 3 .

Conclui-se, portanto, que a opção de compra de mais uma máquina para o subprocesso 4, mesmo sem a consecução do objetivo de alcançar os custos alvo, é a melhor alternativa, por ora. Sugere-se que sejam testados, posteriormente, mudanças mais radicais no processo, uma vez que os valores de MCE indicam um ampla margem de ganho possível. Isto é resultado de layouts inadequados, e/ou de gargalos, que prejudicam o fluxo dos produtos durante a produção, diminuíndo, assim, a capacidade do sistema. Acredita-se que apenas com a diminuição de fatores relacionados à movimentação de materiais, e um melhor balanceamento das máquinas é que haverá um sensivel aumento da capacidade do sistema, reduzindo então os custos de produção aos valores fixados para o custo alvo. 


\section{Bibliografia}

BERLINER, C., BRIMSON, J.A., Gerenciamento de custos em indústrias avançadas, São Paulo, ed. T. A. Queiroz, 1992.

CSILLAG, J.M., Análise do Valor: metodologia do valor: gerenciamento do valor: redução dos custos: racionalização dos custos: racionalização administrativa, 3.ed., São Paulo, ed. Atlas, 1991, 303p.

DAVENPORT, T.H., Reengenharia de Processos, Rio de Janeiro, ed. Campus, 1994.

DRESDEN, M.F., NEUTHE, S., Fertigungsysteme modellieren und simulieren, Zeitschrief für Wirtschaft Fertigung und automatisierung, v.85, n.6, 1990, p.326-329.
PEGDEN,C.D, SHANNON, R.E., SADOWSKI, R. P, Introduction to simulation using SIMAN, McGraw-Hill, Inc, $1990,604 \mathrm{p}$.

ROSS, P.J., Aplicações das técnicas Taguchi na engenharia de qualidade, São Paulo, ed. Makron, 1991.

TEMPELMEIER, H., Konfigurierung flexibler Fertigungsysteme auf dem personal Computer, Zeitschrief für Wirtschaft Fertigung und automatisierung, v.84,n.8,1989, p.448-450.

WITTE,H., HOLLERMANN,C., Simulation Planen der Betriebmittelversorgung, Zeitschrieffür Wirtschaft Fertigung und automatisierung, v.85, n.86, 1990. 